\title{
COHOMOLOGY OF NILRADICALS OF BOREL SUBALGEBRAS
}

BY

\author{
GEORGE F. LEGER AND EUGENE M. LUKS( $(\mathbf{l})$
}

ABSTRACT. Let $\mathbb{R}$ be the maximal nilpotent ideal in a Borel subalgebra of a complex simple Lie algebra. The cohomology groups $H^{\mathcal{L}}(\mathfrak{N}, \mathfrak{R}), H^{1}\left(\mathfrak{R}, \Re^{*}\right)$ and the $\mathbb{R}$-invariant symmetric bilinear forms on $\Re$ are determined. The main result is the computation of $H^{2}(\Re, \Re)$.

1. Introduction. In his well-known paper [2] Professor Kostant has given an algebraic description of the cohomology group $H^{*}(\mathbb{N}, V)$ where $\mathbb{N}$ is the maximal nilpotent ideal of a parabolic subalgebra of a complex simple Lie algebra $g$ and $V$ is an $\mathbb{N}$-module obtained by restriction to $\mathfrak{N}$ of an irreducible $g$-module. In the course of lectures on this and related matters Professor Kostant pointed out the problem of computing the cohomology group $H^{*}(\mathbb{M}, \mathbb{M})$ and stated (indeed it is written in the introduction to [2]) that he knew $H^{1}(\Re, \Re)$ where $\Re$ is the maximal nilpotent ideal of a Borel subalgebra of g. As a result of other work on derivations of Lie algebras we had also obtained $H^{1}(\Re, \Re)$ and we describe this set easily as a consequence of our Theorem 2.4 and Kostant's [2, Theorem 5.14].

The principal goal of this paper is the computation of $H^{2}(\Re, \Re)$. In the course of this we determine $H^{1}\left(\Re, \Re^{*}\right)$ and the $\Re$-invariant symmetric bilinear forms on $\Re$. To facilitate the use of our results we give a description of these groups in such a way that they may be read from the root system. In [4], F. Williams also determines $H^{1}(\Re, \Re)$ and $H^{1}\left(\Re, \Re^{*}\right)$ by different methods which involve extending Kostant's Laplacian.

Throughout this paper $g$ will denote a complex simple Lie algebra with fixed Cartan subalgebra $F_{0}$ We shall assume a fixed linear ordering of the roots with $a_{1}, \cdots, \alpha_{l}$ denoting the simple roots where $l$ is the rank of g, i.e. $l=\operatorname{dim} \xi_{0}$ We shall denote the set of roots by $\Delta$ and the positive roots by $\Delta^{+}$. If $\alpha=\sum_{i=1}^{l} n_{i} \alpha_{i}$ is in $\Delta$ we put $|\alpha|=\sum_{i=1}^{l} n_{i} ;|\alpha|$ is called the level of $a$.

$$
\Re=\sum_{a \in \Delta^{+}} g_{a} ; \quad \Re^{*}=\sum_{a \in \Delta^{+}} g_{-a^{*}}
$$

Thus $g=\Re \oplus \mathscr{G} \oplus \Re^{*}$ and the Killing form of $g$ puts the vector spaces $\Re$, $\Re^{*}$

Received by the editors August 31, 1973.

AMS (MOS) subject classifications (1970). Primary 17B20, 17B30; Secondary 18H25.

(1) This author's research was supported in part by Research Corporation. 
in duality. Further we shall sometimes identify $\Re^{*}$ and $\operatorname{Hom}(\Re, \mathrm{C})$ by means of the Killing form. We choose a fixed element $e_{a}$ from the (one-dimensional) root space of $\alpha$ in such a way that $e_{-\alpha}$ is the dual basis element of $e_{\alpha}$. We note that the $\Re$-module structure induced on $g /(\mathscr{G}+\mathfrak{N})$ by the adjoint representation is the same as the contragredient of the adjoint representation of $\Re$ on itself, i.e.

$$
-t\left(\operatorname{ad} e_{\gamma}\right)\left(\bar{e}_{-a}\right)=\overline{\operatorname{ad} e_{\gamma}\left(e_{-a}\right)}
$$

where $\gamma$ and $\alpha$ are in $\Delta^{+}$. Henceforth we shall use the same symbol $\bar{e}_{-a}$ to denote the coset $e_{-a}+\Re$ in $g / \Re$ or $e_{-a}+(\mathscr{G}+\Re)$ in $g /(\mathscr{g}+\Re)$ since the context will always resolve the ambiguity. As usual, we choose $b_{a}$ in so that $\left(b, b_{a}\right)$ $=a(b)$ for all $b$ in $h$ where $($,$) denotes the Killing form of g$.

2. An exact sequence. The Weyl group to of $g$ is generated by $s_{a_{1}}, \ldots$, $S_{a_{l}}$, the reflections in the simple roots, where

$$
s_{a_{i}}(\beta)=\beta-\frac{2\left(a_{i}, \beta\right)}{\left(a_{i}, \alpha_{i}\right)} a_{i}
$$

and $($,$) denotes the Killing form of g. If s=S_{a_{i_{1}}} \ldots S_{a_{i_{j}}}$ is any element of i then this decomposition is called reduced if any decomposition of $s$ must involve at least $j$ reflections. If this is the case one defines the length $l(s)=j$. Next we let $\Phi_{s}=\left\{\beta \in \Delta^{+} \mid s^{-1}(\beta)<0\right\}$. If $n(s)$ is the cardinality of $\Phi_{s}$ then one has $n(s)=l(s)$. (For this see [1, especially Corollary 3, p. 158].) Further one sees easily from $(*)$ that if $\alpha=\sum_{i=1}^{l} n_{i} \alpha_{i}$ is in $\Delta^{+}$where the $\alpha_{i}$ are simple and the $n_{i}$ are in $\mathrm{Z}^{+}$and if $s(\alpha)<0$ with $s \in \mathbb{Q}$, then a reduced expression for $s$ must have $S_{a_{i}}$ as a factor for $i$ for which $n_{i} \neq 0$. Also one knows that if $\lambda=$ $\sum_{i=1}^{l} n_{i} a_{i}$ is the highest root of $g$ then $n_{i} \neq 0$ for every $1 \leq i \leq l$. Thus we have

2.1. Lemma. Let $\lambda$ denote the bighest root in $\Delta^{+}$and $s$ an element of $\mathcal{C}$. If $n(s)<l$ then $s(\lambda)>0$.

If $0 \rightarrow \Re \rightarrow g \rightarrow g / \Re \rightarrow 0$ is the exact sequence of $\Re$-modules arising from the adjoint representation of $\Re$ on $g$ we have the usual long exact cohomology sequence

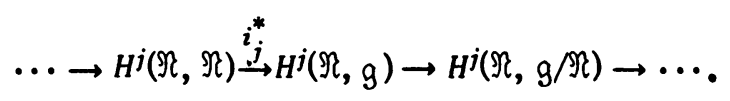

2.2 Theorem. If $l>j$ where $l=\operatorname{rank} g$ then $i_{j}^{*}$ is surjective.

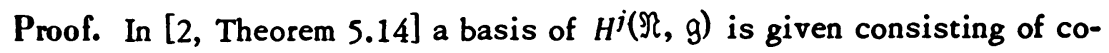
homology classes of cocycles of the form 


$$
e^{*} \otimes e_{s \lambda}, \quad s \in[Q, l(s)=j
$$

where $e_{\Phi_{s}}^{*}=e_{\sigma_{1}}^{*} \wedge \ldots \wedge e_{\sigma_{j}}^{*}$ with $\sigma_{1}<\sigma_{2}<\ldots<\sigma_{j}$ the ordered elements of $\Phi_{s}$ and $\lambda$ is the highest root in $\Delta^{+}$. Such a cocycle is in the image $i_{j}^{*}\left(Z^{j}(\Re, \Re)\right)$ provided $e_{s \lambda}$ is in $\Re$, i.e. if $s \lambda>0$. Thus the theorem follows at once from Lemma 2.1 and the remarks preceding it.

Remark. If $g$ is of type $G_{2}$ one checks that an element of the Weyl group which maps the highest root into a negative root must have length at least three. Thus $i_{2}^{*}$ is also surjective in this case.

2.3 Corollary. If $g$ is not of type $A_{1}, A_{2}$ or $B_{2}$ the sequence

$$
0 \rightarrow H^{1}(\Re, g / \Re) \rightarrow H^{2}(\Re, \Re) \rightarrow H^{2}(\Re, g) \rightarrow 0
$$

is exact.

The following is now immediate.

2.4 Theorem. If $l=$ rank $g>j$ then the sequence

$$
0 \rightarrow H^{j-1}(\Re, g / \Re) \rightarrow H^{j}(\Re, \Re) \rightarrow H^{j(\Re, g) \rightarrow 0}
$$

is exact.

3. Computation of $H^{1}(\Re, \Re)$. In this section we assume $g$ is not of type $A_{1}$, for in that case $\Re$ is one-dimensional and $\operatorname{dim} H^{1}(\Re, \Re)=1$.

Since $H^{0}(\Re, g / \Re)=H^{0}\left(\Re, \mathscr{G}+\Re^{*}\right) \approx \mathscr{K}$, Theorem 2.4 yields the exact sequence

$$
0 \rightarrow \mathfrak{G} \rightarrow H^{1}(\Re, \Re) \rightarrow H^{1}(\Re, g) \rightarrow 0
$$

For $s \in \mathbb{W}, l(s)=1$ if and only if $s=s_{a}$ for some simple root $a$. Thus, by Kostant's description of $H^{*}(\Re, g)$ we see that a basis of $H^{1}(\Re, g)$ is represented by cocycles of the form $e_{-a} \otimes e_{S_{a}(\lambda)}$. This together with the above exact sequence gives

3.1 Theorem. $\operatorname{Dim} H^{1(\Re, \Re)}=2 \times$ rank of $g$.

3.2 Description of the cocycles. (i) If $\alpha_{i}$ is any simple root let $D_{a_{i}}$ denote that derivation of $\Re$ such that

$$
D_{a_{i}}\left(e_{\Sigma_{n_{j}} a_{j}}\right)=n_{i} e_{\Sigma_{n_{j}} a_{j}}
$$

Note that $D_{a_{i}}$ is a scalar multiple of the derivation of $\Re$ induced by $\operatorname{ad}\left(b_{a_{i}}\right)$.

(ii) Let $\lambda$ denote the highest root of $g$. If $\alpha$ is a simple root in $\Delta^{+}$define $D_{\alpha}^{\prime}$ as that derivation of $\Re$ for which

$$
\begin{aligned}
D_{\alpha}^{\prime}\left(e_{\beta}\right) & =e_{S_{\alpha}(\lambda)} & & \text { if } \beta=\alpha, \\
& =0 & & \text { otherwise. }
\end{aligned}
$$


Note that $S_{\alpha}(\lambda)$ is the lowest root of the form $\lambda-q \alpha$.

A basis for $H^{1}(\Re, \Re)$ is given by the cohomology classes of the set of cocycles $D_{a}, D_{\alpha}^{\prime}$ where a runs through the simple roots.

4. A general result on $H^{1}\left(L, L^{*}\right)$. Let $L$ be a finite-dimensional Lie algebra over an arbitrary field $\Phi$. Let $L^{*}$ denote the dual space and $B(L)$ the $L$-module of symmetric bilinear forms on $L$. For a linear transformation, $T$, of $L$ we define

$$
T_{\sharp}: \mathfrak{B}(L) \rightarrow C^{1}\left(L, L^{*}\right)
$$

via $\left(T_{\#} f(x)(y)=f(T x, y)\right.$ for $x, y \in L$. Define

$$
T_{u}: C^{1}\left(L, L^{*}\right) \rightarrow C^{2}(L, \Phi)
$$

so that $T_{\cup} g$ is the cup product $g \cup T$, that is $\left(T_{\cup} g\right)(x, y)=g(x)(T y)-g(y)(T x)$. Then $T_{U} \circ T_{\#}=0$. If $T$ is a derivation of $L$ then $T_{\#}\left(\mathscr{B}(L)^{L}\right) \subset Z^{1}\left(L, L^{*}\right)$, $T_{U}\left(Z^{1}\left(L, L^{*}\right)\right) \subset Z^{2}(L, \Phi)$ and we have induced maps

$$
T_{\#}^{*}: \mathfrak{B}(L)^{L} \rightarrow H^{1}\left(L, L^{*}\right), \quad T_{\cup}^{*}: H^{1}\left(L, L^{*}\right) \rightarrow H^{2}(L, \Phi) .
$$

4.1 Theorem. Suppose $L$ bas a derivation $T$ such that $I_{L} \otimes T+T \otimes I_{L}$ is a nonsingular transformation of $L \otimes L$. Then the sequence

$$
0 \rightarrow \mathcal{B}(L)^{L} \stackrel{T_{\sharp}^{*}}{\rightarrow} H^{1}\left(L, L^{*}\right) \stackrel{T_{u}^{*}}{\rightarrow} H^{2}(L, \Phi) \rightarrow 0
$$

is exact.

Proof. We remark that the nonsingularity of $I_{L} \otimes T+T \otimes I_{L}$ is equivalent to the hypothesis that the sum of two characteristic roots of $T$ is never 0 . In particular, $T$ is nonsingular on $L$. It is useful to consider the transpose, $\hat{T}$, of $I_{L} \otimes T+T \otimes I_{L}:$

$$
\hat{T}:(L \otimes L)^{*} \rightarrow(L \otimes L)^{*}
$$

Note that $\hat{T}$ preserves the subspaces $B(L)$ and $C^{2}(L, \Phi)$ of symmetric and skewsymmetric functions, respectively. Within these $B(L)^{L}, Z^{2}(L, \Phi)$ and $B^{2}(L, \Phi)$ are also preserved. (For $B^{2}(L, \Phi)$, note $\hat{T}(\delta a)=\delta(a \circ T)$ ). Thus, since $\hat{T}$ is nonsingular, $f$ is in one of these subspaces if and only if $\hat{T} f$ is.

The theorem follows from the following three observations:

(1) The sequence

$$
0 \rightarrow \mathfrak{B}(L)^{L} \stackrel{T_{\sharp}}{\rightarrow} Z^{1}\left(L, L^{*}\right) \stackrel{T_{u}}{\rightarrow} Z^{2}(L, \Phi) \rightarrow 0
$$

is exact.

(2) $T_{\#}\left(B(L)^{L}\right) \cap B^{1}\left(L, L^{*}\right)=0$. 
(3) $T_{U}\left(B^{1}\left(L, L^{*}\right)\right)=B^{2}(L, \Phi)$.

Proof of (1). $T_{\#}$ is injective since $T$ is surjective. As already noted $\operatorname{im}\left(T_{\#}\right) \subset \operatorname{ker}\left(T_{U}\right)$. Let $g \in \operatorname{ker}\left(T_{U}\right) \cap Z^{1}\left(L, L^{*}\right)$. Then, for $x, y \in L, g(x)(T y)=$ $g(y)(T x)$. Thus, if $f(x, y)=g\left(T^{-1} x\right)(y)$ for $x, y \in L, f$ is in $B(L)$ and $T_{\#} f=g$. To show $f \in \mathscr{B}(L)^{L}$ it suffices to show $\hat{T} f \in \mathfrak{B}(L)^{L}$. But $\delta g=0$ yields, for all $x, y, z \in L$

$$
0=\delta g(x, y)(z)+\delta g(x, z)(y)=(\hat{T} f)([y, x], z)+(\hat{T} f)(y,[z, x])=(x \cdot \hat{T} f)(y, z) .
$$

To show $T_{U}\left(Z^{1}\left(L, L^{*}\right)\right)=Z^{2}(L, \Phi)$, we take $b \in Z^{2}(L, \Phi)$. Let $b^{\prime}=\hat{T}^{-1}(b)$ and define $g \in C^{1}\left(L, L^{*}\right)$ by $g(x)(y)=b^{\prime}(x, y)$. Since $b^{\prime} \in Z^{2}(L, \Phi),(\delta g)(x, y)(z)=$ $\delta b^{\prime}(x, y, z)=0$. Thus $g \in Z^{1}\left(L, L^{*}\right)$ and

$$
\left(T_{1, g}\right)(x, y)=b^{\prime}(x, T y)+b^{\prime}(T x, y)=b(x, y) .
$$

Hence (1) is proved.

Proof of (2). Take $g$ in $C^{1}\left(L, L^{*}\right)$ such that $g=\delta a$ with $a \in L^{*}=C^{0}\left(L, L^{*}\right)$. Then $g(x)=x \cdot a$ for all $x$ in $L$ and so $g(x)(y)=-a([x, y])$ for $x, y \in L$. Now if $g \in \operatorname{im}\left(T_{\#}\right) \subset \operatorname{ker}\left(T_{U}\right)$ then

$$
0=\left(T_{u} g\right)(x, y)=g(x)(T y)-g(y)(T x)=-a([x, T y]-[y, T x])=-a(T[x, y]) .
$$

But $T$ is nonsingular, so $T\left(L^{2}\right)=L^{2}$. Thus $g=0$.

Proof of (3). Let $\delta a \in B^{2}(L, \phi)$ where $a \in C^{1}(L, \Phi)=L^{*}$. Let $b=a \circ T^{-1}$. Considering $b$ as an element of $C^{0}\left(L, L^{*}\right)$ one has $T_{U}(\delta b)=\delta a$.

5. Computation of $H^{1}\left(\Re, \Re^{*}\right)$. We shall apply Theorem 4.1 to $\Re$. Let $T$ be the linear transformation of $\Re$ such that $T e_{\sigma}=|\sigma| e_{\sigma}$ for $\sigma \in \Delta^{+}$where $|\sigma|$ denotes the level of the root $\sigma$. Then $T$ is a (diagonal) derivation of $\Re$ with only positive eigenvalues and so it satisfies the hypothesis of Theorem 4.1. Thus we turn our attention to $\mathfrak{B}(\Re)^{\Re}$.

5.1 Theorem. $B(\Re)^{\Re}=\left\{f \in \mathscr{B}(\Re) \mid f\left(e_{\sigma}, e_{\tau}\right)=0\right.$ if $\sigma$ or $r$ is not simple $\}$

Before proving the above we need

5.2 Lemma. Suppose $\alpha, \beta, \rho$ are distinct roots in $\Delta^{+}$with $\alpha, \beta$ simple and such that $\alpha+\beta, \rho+\alpha, \rho+\beta$ are also in $\Delta$. Then

(i) $\alpha+2 \beta \notin \Delta$ and $\rho-\beta \in \Delta$, or

(ii) $\beta+2 \alpha \notin \Delta$ and $\rho-\alpha \in \Delta$.

Proof. Recall that the string of roots of the form

$$
\tau-r \sigma, r-(r-1) \sigma, \cdots, \tau, \tau+\sigma, \cdots, r+q \sigma
$$

with $q, r$ nonnegative integers is called the $\sigma$ string containing $r$. If $($,$) de-$ notes the Killing form of $g$ then $2(\sigma, r) /(\sigma, \sigma)=r-q$. It is well known that $r+q \leq 3$. 
(1) $\alpha+2 \beta \notin \Delta$ or $2 \alpha+\beta \notin \Delta$.

For this, $0<(\alpha+\beta, \alpha+\beta)=(\alpha+\beta, \alpha)+(\alpha+\beta, \beta)$ and $(\alpha+\beta, \alpha)>0$ implies $2 \alpha+\beta \notin \Delta$ since $\alpha-\beta \notin \Delta$ while $(\alpha+\beta, \beta)>0$ implies $\alpha+2 \beta \notin \Delta$.

(2) $\rho-\beta \notin \Delta$ implies $\rho+\alpha+2 \beta$ is in $\Delta$. For this

$$
\frac{2(\rho+\alpha, \beta)}{(\beta, \beta)}=\frac{2(\alpha, \beta)}{(\beta, \beta)}+\frac{2(\rho, \beta)}{(\beta, \beta)} \leq-1-1=-2
$$

Thus the roots $\rho+\alpha, \rho+\alpha+\beta, \rho+\alpha+2 \beta$ are in $\Delta$.

(3) $\rho-\beta \in \Delta$ or $\rho-\alpha \in \Delta$.

If false, i.e. if $\rho-\beta \notin \Delta$ and $\rho-\alpha \notin \Delta$, then $2(\rho, \alpha) /(\alpha, a) \leq-1$. By (2), $\rho+\alpha+2 \beta \in \Delta$ and

$$
\frac{2(\rho+\alpha+2 \beta, \alpha)}{(\alpha, \alpha)}=\frac{2(\rho, \alpha)}{(\alpha, \alpha)}+2+2 \cdot \frac{2(\beta, \alpha)}{(\alpha, \alpha)} \leq-1+2-2=-1
$$

so $\Delta$ would have the roots $\rho+\alpha+2 \beta, \rho+2 \alpha+2 \beta$. Since $\sigma \in \Delta$ implies $2 \sigma \notin \Delta$, $\rho$ can neither be added to nor subtracted from $\rho+2 \alpha+2 \beta$ with the result in

$\Delta$. Thus the contradiction

$$
0=\frac{(\rho, \rho+2 \alpha+2 \beta)}{(\rho, \rho)}=1+\frac{2(\rho, \alpha)}{(\rho, \rho)}+\frac{2(\rho, \beta)}{(\rho, \rho)} \leq-1 .
$$

Now suppose the lemma false so that, by (1) and (3) (after possibly interchanging $\alpha, \beta)$ we have

$$
\alpha+2 \beta \notin \Delta, \quad 2 a+\beta \epsilon \Delta ; \quad \rho-\beta \notin \Delta, \quad \rho-\alpha \epsilon \Delta .
$$

Since $\alpha, \alpha+\beta, 2 a+\beta \in \Delta, 2(\alpha, \beta) /(\alpha, \alpha) \leq-2$ or $(\alpha, \beta) \leq-(\alpha, \alpha)$. Since $2 a$, $2 \rho \notin \Delta, 0=(\rho-\alpha, \rho+\alpha)$, or $(\rho, \rho)=(\alpha, \alpha)$. Now, from (2), $\rho+\alpha+\beta$ and $\rho+$ $\alpha+2 \beta$ are in $\Delta$. Thus

$$
\begin{aligned}
0 & =(\rho+\alpha, \rho+\alpha+2 \beta)=(\rho, \rho)+2(\rho, \alpha)+(\alpha, \alpha)+2(\beta, \rho)+2(\alpha, \beta) \\
& \leq(\alpha, \alpha)+2(\rho, \alpha)+(\alpha, \alpha)+2(\beta, \rho)-2(\alpha, \alpha)=2(\alpha+\beta, \rho) .
\end{aligned}
$$

Thus the $\alpha+\beta$ string containing $\rho$ cannot contain $\rho+2(\alpha+\beta)$, i.e. $\rho+2 \alpha+2 \beta \notin \Delta$.

$$
0 \leq(\rho+\alpha+2 \beta, \alpha)=(\rho, \alpha)+(\alpha, \alpha)+2(\beta, \alpha) \leq(\rho, \alpha)-(\alpha, \alpha)
$$

or

$$
\frac{2(\rho, a)}{(a, \alpha)} \geq 2
$$

This is impossible since $\rho+a \in \Delta$.

5.3 Remark. The statement of Lemma 5.2 is similar to an observation made about $\Delta^{+}$in [3, Remark 5.2] and the latter is, in fact, an easy corollary of the 
present statement. These properties were discovered by direct investigation of root tables. We take this opportunity to thank Professor T. A. Springer for suggesting the possibility of a unified proof.

It is interesting to note, as shown in [3], one direct consequence of Lemma 5.2 is that $H^{2}(\Re, \Re)^{5}=0$. Here we shall see that the lemma leads to the determination of $\mathcal{B}(\Re)^{\Re}$ and ultimately to further information about $H^{2}(\Re, \Re)$.

We come now to the

Proof of Theorem 5.1. $\mathfrak{B}(\Re)$ is canonically isomorphic to $\left(\Re_{(} \Re\right)^{*}$ where (S)

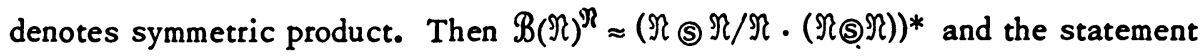
of the theorem translates to

$$
\Re\left(5 \Re^{2}=\Re \cdot(\Re(5) \text {. }\right.
$$

Since $\Re(5) \Re^{2}$ is spanned by the products $e_{\sigma}(5) e_{\tau}$ where $\sigma, \tau \in \Delta^{+}$and $\max (|\sigma|,|\tau|)>1$, it suffices to show that

$$
e_{\sigma}\left(S e _ { \tau } \in \Re \cdot \left(\Re(\text { (S) }) \text { for } \sigma, \tau \in \Delta^{+} \text {and } \max (|\sigma|,|r|)>1\right.\right. \text {. }
$$

We proceed by backward induction on $\max (|\sigma|,|\tau|)$.

If $|\tau|$ is maximal write $c e_{\tau}=\left[e_{\alpha}, e_{\mu}\right]$ with $c \neq 0$ and $a$ simple. Then

$$
e_{\mu} \cdot\left(e_{\sigma}\left(e_{\alpha}\right)=\left[e_{\mu}, e_{\sigma}\right] \text { (S) } e_{\alpha}-c\left(e_{\sigma}\left(5 e_{\tau}\right)\right.\right.
$$

so that we are cione if $\left[e_{\mu}, e_{\sigma}\right]=0$. If $\left[e_{\mu}, e_{\sigma}\right] \neq 0, \sigma$ must be simple and $\mu+\sigma$ $=r=\mu+\alpha$ so that $\sigma=\alpha$. Then $e_{\mu} \cdot\left(e_{\sigma}\right.$ (S) $\left.e_{\alpha}\right)=-2 c\left(e_{\sigma}\right.$ (5) $\left.e_{\tau}\right)$ and the result is proved in this case.

Now suppose the result holds for $\max (|\sigma|,|\tau|)>k$ and that $|\sigma| \leq|\tau|=k$. If $\sigma$ is not simple $c e_{\sigma}=\left[e_{\alpha}, e_{\delta}\right]$ with $a$ simple, $c \neq 0$ so that

$$
e_{\alpha} \cdot\left(e_{\delta}\left(e_{\tau}\right)=c e_{\sigma} @ e_{\tau}+e_{\delta} \leqq\left[e_{a}, e_{\tau}\right]\right. \text {. }
$$

$e_{\delta}\left(5\left[e_{a}, e_{\tau}\right]\right.$ is in $\Re$. ( $\left(\right.$ (S) $\left.\Re^{*}\right)$ by the induction hypothesis and so we are done if $\sigma$ is not simple.

We suppose now that $\sigma$ is simple. Then $\tau$ cannot be simple so we may write $c e_{r}=\left[e_{a}, e_{\mu}\right]$ with $a$ simple and $c \neq 0$. We have

(i) $e_{\alpha} \cdot\left(e_{\sigma}\right.$ (S) $\left.e_{\mu}\right)=\left[e_{\alpha}, e_{\sigma}\right]$ (S) $e_{\mu}+c\left(e_{\sigma}\right.$ (S $\left.e_{\tau}\right)$, and

(ii) $e_{\mu} \cdot\left(e_{\sigma}\right.$ (s) $\left.e_{\alpha}\right)=\left[e_{\mu}, e_{\sigma}\right]$ (s) $e_{\alpha}-c\left(e_{\sigma}\right.$ (s) $\left.e_{\tau}\right)$.

Thus we are done if either $\alpha+\sigma \notin \Delta$ or $\mu+\sigma \notin \Delta$. So we may assume

$$
\alpha+\sigma \in \Delta, \quad \mu+\sigma \in \Delta, \quad \tau=\alpha+\mu \epsilon \Delta .
$$

By Lemma 5.1 either (a) $\sigma+2 \alpha \notin \Delta$ and $\mu-a \epsilon \Delta$, or (b) $2 \sigma+a \notin \Delta$ and $\mu-\sigma \in \Delta$.

In case (a) $c^{\prime}\left[e_{\alpha}, e_{\sigma}\right]$ (s) $e_{\mu}=e_{\alpha} \cdot\left(\left[e_{\alpha}, e_{\sigma}\right]\right.$ (s) $\left.e_{\mu-\alpha}\right)$ with $c^{\prime} \neq 0$ so that we are done by (i). 

proof.

In case (b) $c^{\prime \prime}\left[e_{\mu}, e_{\sigma}\right] \odot e_{\alpha}=e_{\sigma} \cdot\left(\left[e_{\alpha}, e_{\sigma}\right] \Theta e_{\mu-\sigma}\right)$ and (ii) finishes the

With $T\left(e_{\sigma}\right)=|\sigma| e_{\sigma}$, Theorem 4.1 and 5.1 yield

5.4 Theorem. The sequence

$$
0 \rightarrow \delta \stackrel{i^{*}}{\rightarrow} H^{1}\left(\Re, \Re^{*}\right) \stackrel{T_{u}^{*}}{\rightarrow} H^{2}(\Re, \mathrm{C}) \rightarrow 0
$$

is exact, where $\delta=\left\{f \in \operatorname{Hom}\left(\Re, \Re^{*}\right) \mid f\left(e_{\alpha}\right)\left(e_{\beta}\right)=f\left(e_{\beta}\right)\left(e_{\alpha}\right)\right.$ and $f\left(e_{\sigma}\right)\left(e_{\tau}\right)=0$ if either $\sigma$ or $\tau$ is not simple $\}$ and $i^{*}$ is induced by the injection $i: \delta \rightarrow \operatorname{Hom}\left(\Re, \Re^{*}\right)$.

In particular, $\operatorname{dim} H^{1}\left(\Re, \Re^{*}\right)=1 / 2 l(l+1)+1 / 2(l+2)(l-1)=l^{2}+l-1$.

5.5 Description of the cocycles. It is clear how $\delta$ is embedded in $H^{1}(\Re, \Omega * *)$. For the reader's convenience we have traced the embedding of $H^{2}(\Re, \mathrm{C})$ in $H^{1}\left(\Re, \Re^{*}\right)$. We first explicate the basis of $H^{2}(\Re, C)$ given in $[2,5.14]$. Let $\alpha, \beta$ be distinct simple roots in $\Delta^{+}$and let $\alpha+r \beta$ be the highest root in the $\beta$ string containing $a$. Define $b_{\alpha \beta} \in Z^{2}(\Re, C)$ so that

$$
\begin{aligned}
b_{\alpha \beta}\left(e_{\sigma}, e_{\tau}\right)=1 & \text { if } \sigma=\alpha+r \beta, \tau=\beta, \\
& =0 \quad \text { if }\{\sigma, \tau\} \neq\{\alpha+r \beta, \beta\} .
\end{aligned}
$$

Observe that $b_{a \beta}=-b_{\beta a}$ if $a+\beta \notin \Delta^{+}$. Taking any ordering, $<$, of the simple roots, a basis of $H^{2}(\Re, C)$ is represented by the functions

$$
\left\{b_{a \beta} \mid a+\beta \epsilon \Delta \text { or } \alpha<\beta \text { and } \alpha+\beta \notin \Delta\right\} \text {. }
$$

As in the proof of Theorem $4.1, b_{\alpha \beta}$ gives rise to $g_{\alpha \beta} \in Z^{1}\left(\Re, \Re^{*}\right)$ where

$$
\begin{aligned}
g_{\alpha \beta}\left(e_{\sigma}\right)\left(e_{r}\right) & =1 & & \text { if } \sigma=\alpha+r \beta, r=\beta, \\
& =-1 & & \text { if } \sigma=\beta, r=\alpha+r \beta, \\
& =0 & & \text { otherwise. }
\end{aligned}
$$

(Actually, with $T e_{\sigma}=|\sigma| e_{\sigma}$ as before, this $g_{\alpha \beta}$ is $r+2$ times the function constructed in the proof.) Then a basis for the image of $H^{2}(\Re, C)$ in $H^{1}\left(\Re, \Re^{*}\right)$ is represented by $\left\{g_{\alpha \beta} \mid a+\beta \in \Delta\right.$ or $\alpha<\beta$ and $\left.\alpha+\beta \in \Delta\right\}$.

6. Computation of $H^{1}(\Re, g / \Re)$ and $H^{2}(\Re, \Re)$. We write $g / \Re=\xi+\Re *$, thus regarding $\bar{G}$ as a trivial $\Re$-module. If $f$ is an element of $\operatorname{Hom}\left(\Re, \Re^{*}\right)$ we may write

$$
f\left(e_{\sigma}\right)=\sum_{r \in \Delta^{+}} a_{\sigma \tau} \bar{e}_{-\tau} \text { for } \sigma \in \Delta^{+}
$$

Then $f$ is in the subspace $\delta$ of "symmetric maps" (as in Theorem 5.4) provided $a_{\sigma \tau}=a_{\gamma \sigma}$ and $a_{\sigma \tau}=0$ if either $\sigma$ or $r$ is not simple. Let

$$
\begin{aligned}
& \mathcal{H}_{0}=\left\{f \in \operatorname{Hom}\left(\Re, \Re^{*}\right) \mid a_{\sigma \tau}=0 \text { when } \sigma \neq \tau \text { and } \sigma+\tau \notin \Delta\right\} \\
& \mathcal{H}_{1}=\left\{f \in \operatorname{Hom}\left(\Re, \Re^{*}\right) \mid a_{\sigma \tau}=0 \text { when } \sigma=\tau \text { or } \sigma+\tau \in \Delta\right\} .
\end{aligned}
$$


Note that the basis given in 5.5 representing the image of $H^{2}(\Re, \mathrm{C})$ in $H^{1}\left(\Re, \Re^{*}\right)$ is contained in $\mathcal{H}_{1}$ (by the choice of $r, a+r \beta+\beta \notin \Delta$ ). Now let

$$
\mathcal{S}_{0}=\mathcal{S} \cap \mathcal{H}_{0}, \quad \mathcal{S}_{1}=\mathcal{S}_{1} \mathcal{H}_{1}
$$

Clearly $\delta=S_{0} \oplus S_{1}$.

Let $W=\Sigma_{a \text { simple }}\left(e_{\alpha}\right)$. Denote by $D$ the subspace of $\operatorname{Hom}(W, G)$ consisting of maps $d$ such that $d\left(e_{a}\right) \subset\left(b_{a}\right)$.

6.1 Theorem. $H^{1}(\Re, g / \Re) \approx \delta_{0} \oplus \operatorname{Hom}(W, \mathfrak{G}) / D$. In particular,

$$
\operatorname{dim}\left(H^{1}(\Re, g / \Re)\right)=(2 l-1)+l^{2}-l=l^{2}+l-1 .
$$

Proof. We have the exact cohomology sequence

$$
H^{0}\left(\Re, \Re^{*}\right) \stackrel{\delta_{0}^{*}}{\rightarrow} H^{1}(\Re, \mathfrak{\zeta}) \stackrel{i^{*}}{\rightarrow} H^{1}\left(\Re, \mathfrak{G}+\Re^{*}\right) \stackrel{j^{*}}{\rightarrow} H^{1}\left(\Re, \Re^{*}\right) \stackrel{\delta_{1}^{*}}{\rightarrow} H^{2}(\Re, \mathfrak{\zeta}) .
$$

Note first that $H^{1}(\Re, \zeta) \approx \operatorname{Hom}\left(\Re / \Re^{2}, \zeta\right) \approx \operatorname{Hom}(W, \zeta)$ and $H^{0}\left(\Re, \Re^{*}\right) \approx$ $\Sigma_{a \text { simple }}\left(e_{-\alpha}\right)$. With these identifications $\delta_{0}^{*}\left(e_{-\alpha}\right)$ is the map induced by ad $\left(e_{\alpha}\right)$. Thus we see $\operatorname{im}\left(\delta_{0}^{*}\right)=D$ and so $\operatorname{im}\left(i^{*}\right) \approx \operatorname{Hom}\left(W, L_{)}\right) / D$. We then have a short exact sequence

$$
0 \rightarrow \operatorname{Hom}(W, \mathfrak{\zeta}) / D \rightarrow H^{1}\left(\Re, \zeta+\Re^{*}\right) \rightarrow \operatorname{ker}\left(\delta_{1}^{*}\right) \rightarrow 0 .
$$

It remains to show that $\operatorname{ker} \delta_{1}^{*} \approx \mathfrak{S}_{0}$.

If $f \in Z^{1}\left(\Re, \Re^{*}\right)$, we may lift $f$ to $\hat{f}: \Re \rightarrow \mathfrak{G}+\Re^{*}$ so that $\operatorname{im}(f) \subset \Re^{*}$. The image of the cohomology class of $f$ under $\delta_{1}^{*}$ is represented by $F \in C^{2}(\Omega, \zeta)$ such that

$$
F\left(e_{\sigma}, e_{\tau}\right)=\delta \hat{f}\left(e_{\sigma}, e_{\tau}\right)=\left(e_{\sigma} \cdot \hat{f}\right)\left(e_{\tau}\right)-\left(e_{\tau} \cdot \hat{f}\right)\left(e_{\sigma}\right)-\hat{f}\left[e_{\sigma}, e_{\tau}\right]
$$

Since im $F \subset \bar{h}$ one has, referring to equation (A)

$$
F\left(e_{\sigma}, e_{\tau}\right)=a_{\alpha \tau}\left[e_{\sigma}, e_{-\sigma}\right]-a_{\tau \sigma}\left[e_{\tau}, e_{-\tau}\right] .
$$

Suppose $f \epsilon \delta_{0}$. Then $F\left(e_{\sigma}, e_{\tau}\right)=0$ unless $\sigma$ and $\tau$ are simple and $\sigma+\tau \epsilon$ $\Delta$. Then we may define $g \in C^{1}(\Re, \mathfrak{h})$ so that

$$
g\left(\left[e_{\sigma}, e_{\tau}\right]\right)=-F\left(e_{\sigma}, e_{\tau}\right) \text { for all } \sigma, \tau \in \Delta^{+} .
$$

Then $F=\delta g$. Thus $\delta_{1}^{*}\left(\delta_{0}\right)=0$.

It is now sufficient to prove that, if $f$ represents a cohomology class in $\operatorname{ker}\left(\delta_{1}^{*}\right)$ then $f \in \mathcal{H}_{0}$. For then $\left(\delta_{1} \oplus H^{2}(\Re, \mathrm{C})\right) \cap \operatorname{ker}\left(\delta_{1}^{*}\right)=0$ and the proof is complete by Theorem 5.4. Choose such an $f$. With $F$ as before, there must be some $g \in C^{1}(\Re, \mathfrak{G})$ so that $\delta g=F$. By equation (B)

$$
-g\left(\left[e_{\sigma}, e_{\tau}\right]\right)=a_{\sigma \tau}\left[e_{\sigma}, e_{-\sigma}\right]-a_{\tau \sigma}\left[e_{\tau}, e_{-\tau}\right] \text { for } \sigma, \tau \in \Delta \text {. }
$$


If $\sigma+\tau \notin \Delta,\left[e_{\sigma}, e_{\tau}\right]=0$ and so $0=a_{\sigma \tau}\left[e_{\sigma}, e_{-\sigma}\right]-a_{\tau \sigma}\left[e_{\tau}, e_{-\tau}\right]$. The result follows now from:

6.2 Lemma. If $\sigma$ and $\tau$ are distinct elements of $\Delta^{+}$then $\left[e_{\sigma}, e_{-} \sigma\right]$ and $\left[e_{\tau}, e_{-\tau}\right]$ are linearly independent.

Proof. Recall that $b_{\beta}$ is chosen in $\bar{g}$ so that $\left(b, b_{\beta}\right)=\beta(b)$ for all $b$ in $\xi_{0}$ Clearly, if $\sigma, \tau, \sigma+\tau$ are in $\Delta^{+}$then $b_{\sigma+\tau}=b_{\sigma}+b_{\tau^{*}}$. Thus if $\sigma=\Sigma n_{a} \alpha$ with $n_{a}$ $\geq 0$, a simple, $b_{a}=\Sigma n_{\alpha} b_{a}$. Since $\left[e_{\sigma}, e_{-\sigma}\right]$ is a scalar multiple of $b_{\sigma}$ the lemma follows.

6.3 Description of the cocycles. It is clear how an element of $\mathrm{Hom}(W, \zeta) / D$ gives rise to a class in $H^{1}\left(\Re, \zeta+\Re^{*}\right)$. However the proof did not explicitly determine $j\left(\mathcal{S}_{0}\right)$. We now indicate a basis of this set.

$\delta_{0}$ is spanned by the functions $f_{\alpha}$, for $\alpha$ simple, and $f_{\{\alpha, \beta\}}$, for $\alpha, \beta$ simple and $\alpha+\beta \in \Delta^{+}$where

$$
f_{a}\left(e_{\sigma}\right)=\left\{\begin{array} { l l } 
{ \overline { e } _ { - \alpha } } & { \text { if } \sigma = a , } \\
{ 0 } & { \text { otherwise. } }
\end{array} \quad \left\{\{a, \beta\}\left(e_{\sigma}\right)= \begin{cases}\bar{e}_{-\beta} & \text { if } \sigma=a, \\
\bar{e}_{-\alpha} & \text { if } \sigma=\beta, \\
0 & \text { otherwise. }\end{cases}\right.\right.
$$

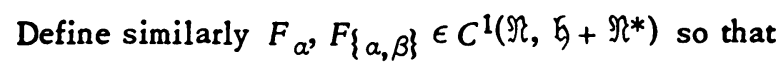

$$
\begin{aligned}
& F_{\alpha}\left(e_{\sigma}\right)= \begin{cases}\bar{e}_{-\alpha} & \text { if } \sigma=a, \\
0 & \text { otherwise. }\end{cases} \\
& F_{\{a, \beta\}}\left(e_{\sigma}\right)= \begin{cases}\bar{e}_{-\beta} \text { if } \sigma=\alpha, \\
\bar{e}_{-\alpha} \text { if } \sigma=\beta, \\
c\left(\left[e_{\alpha}, e_{-\alpha}\right]-\left[e_{\beta}, e_{-\beta}\right]\right) & \text { if } \sigma=\alpha+\beta \\
0 \quad \text { otherwise. } & \text { where } e_{\sigma}=c\left[e_{\alpha}, e_{\beta}\right],\end{cases}
\end{aligned}
$$

Then $F_{a}, F_{\{a, \beta\}} \in Z^{1}\left(\Re, \mathscr{G}+\Re^{*}\right)$ and their classes in $H^{1}\left(\Re, \mathscr{G}+\Re^{*}\right)$ map by $j^{*}$ to those of $f_{a}, f_{\{a, \beta\}}$, respectively.

By Corollary 2.3 and Theorem 6.1

6.4 Theorem. If $g$ is not of type $A_{1}, A_{2}$ or $B_{2}$, then

$$
H^{2}(\Re, \Re) \approx H^{2}(\Re, \zeta) \oplus S_{0} \oplus \operatorname{Hom}(W, \zeta) / D .
$$

In particular, $\operatorname{dim} H^{2}(\Re, \Re)=1 / 2(l+2)(l-1)+l^{2}-l-1=1 / 2\left(3 l^{2}+3 l-4\right)$.

6.5 Description of the cocycles. (i) Cocycles representing a basis of $H^{2}(\Re, g)$ have been given by Kostant and his description is given in the proof of Theorem 2.2. To be more explicit: Let $\alpha, \beta$ be distinct simple roots in $\Delta^{+}$. Let 
$\alpha+r \beta$ be the highest root in the $\beta$ string containing $\alpha$, and let $\lambda$ be the highest root in $\Delta^{+}$. Define $K_{\alpha \beta} \in C^{2}(\Re, \Re)$ by

$$
\begin{aligned}
K_{a \beta}\left(e_{\sigma}, e_{\tau}\right) & =S_{\beta} S_{\alpha}(\lambda) & & \text { if } \sigma=\alpha+r \beta, \tau=\beta, \\
& =0 & & \text { if }\{\sigma, \tau\} \neq\{\alpha+r \beta, \beta\} .
\end{aligned}
$$

Note that $S_{\beta} S_{a}(\lambda)$ is the lowest root in the $\beta$ string containing the lowest root in the $\alpha$ string containing $\lambda$. Observe that $K_{a \beta}=-K_{\beta a}$ if $\alpha+\beta \notin \Delta$ (then $S_{\alpha} S_{\beta}=$ $\left.S_{\beta} S_{\alpha}\right)$. Taking any ordering, $<$, of the simple roots, a basis of $H^{2}(\Re, g)$ is represented by the cocycles $\left\{K_{a \beta} \mid \alpha+\beta \in \Delta\right.$ or $\alpha<\beta$ and $\left.\alpha+\beta \notin \Delta\right\}$.

(ii) The image of $\mathcal{S}_{0}$ in $H^{1}(\Re, g / \Re)$ is described in 6.3. The $F_{a}, F_{\{a, \beta\}}$ given there correspond to $G_{a}, G_{\{a, \beta\}} \in Z^{2}(\Re, \Re)$ where:

For a simple

$$
\begin{array}{ll}
G_{\alpha}\left(e_{\alpha}, e_{\sigma}\right)=\left[e_{-\alpha}, e_{\sigma}\right] & \text { for all } \sigma \neq \alpha, \sigma \in \Delta^{+}, \\
G_{\alpha}\left(e_{\sigma}, e_{\tau}\right)=0 & \text { if } \alpha \notin\{\sigma, \tau\} .
\end{array}
$$

For $\alpha, \beta$ simple with $\alpha+\beta \in \Delta$

$$
\begin{array}{ll}
G_{\{a, \beta\}}\left(e_{\alpha}, e_{\sigma}\right)=\left[e_{-\beta}, e_{\sigma}\right] & \text { for } \sigma \neq \beta, \\
G_{\{a, \beta\}}\left(e_{\beta}, e_{\sigma}\right)=\left[e_{-\alpha}, e_{\sigma}\right] & \text { for } \sigma \neq \alpha, \\
G_{\{a, \beta\}}\left(e_{\sigma}, e_{\tau}\right)=0 & \text { for } \sigma, \tau \in \Delta, \sigma \neq \tau, \\
& \text { if }\{\sigma, \tau\} \cap\{\alpha, \beta\} \neq\{\alpha\} \text { or }\{\beta\} .
\end{array}
$$

(iii) If $f$ represents a coset in $\operatorname{Hom}(W, G) / D$ the corresponding class in $H^{2}(\Re, \Re)$ is represented by $F$ where $F\left(e_{\alpha}, e_{\beta}\right)=\left[f\left(e_{\alpha}\right), e_{\beta}\right]-\left[f\left(e_{\beta}\right), e_{\alpha}\right]$ noting that $f\left(e_{\alpha}\right)=0$ if $a$ is not simple.

6.6 Remark. For completeness we describe $H^{2}(\Re, \Re)$ when $\Re$ is of type $A_{2}$ or $B_{2}$.

In the case of $A_{2}$ the images of the highest root in $\Delta^{+}$through the length-2 Weyl group elements are not in $\Delta^{+}$. Hence $\operatorname{Im}\left(H^{2}(\Re, \Re) \rightarrow H^{2}(\Re, g)\right)=0$. Since $H^{1}(\Re, \Re) \rightarrow H^{1}(\Re, g)$ is surjective by Theorem 2.2 , we have $H^{1}(\Re, g / \Re) \approx$ $H^{2}(\Re, \Re)$. By Theorem 6.1, $\operatorname{dim} H^{2}(\Re, \Re)=5$ and representing cocycles are given in (ii), (iii) above.

In the case of $B_{2}, \Delta^{+}=\left\{a_{1}, a_{2}, a_{1}+a_{2}, a_{1}+2 \alpha_{2}\right\}$. Then $S_{a_{1}} s_{a_{2}}\left(a_{1}+2 a_{2}\right)$ $=-a_{1} \notin \Delta^{+}$, while $S_{a_{2}} S_{a_{1}}\left(a_{1}+2 a_{2}\right)=a_{1}$. Hence $\operatorname{Im}\left(H^{2}(\Re, \Re) \rightarrow H^{2}(\Re, g)\right)$ is spanned by the class of the cocycle $K$ where

$$
K\left(e_{a_{2}}, e_{a_{1}+2 a_{2}}\right)=e_{a_{1}} ; \quad K\left(e_{\sigma}, e_{\tau}\right)=0 \text { if }\{\sigma, \tau\} \neq\left\{a_{2}, a_{1}+2 \alpha_{2}\right\} .
$$

Now we have $\operatorname{dim} H^{2}(\Re, \Re)=6$ with representing cocycles $K$ and those given in (ii) and (iii). 


\section{REFERENCES}

1. N. Bourbaki, Eléments de mathématique. Fasc. XXXIV. Groupes et algèbres de Lie. Chap. IV: Groupes de Coxeter et systèmes de Tits. Chap. V: Groupes engendrés par des réflexions. Chap. VI: Systèmes de racines, Actualités Sci. Indust., no. 1337, Hermann, Paris, 1968. MR $39 \# 1590$.

2. B. Kostant, Lie algebra cohomology and the generalized Borel-Weil theorem, Ann. of Math. (2) 74 (1961), 329-387. MR $26 \# 265$.

3. G. Leger and E. Luks, Cohomology theorems for Borel-like solvable Lie algebras in arbitrary characteristic, Canad. J. Math. 24 (1972), 1019-1026.

4. F. Williams, Laplace operators and the b-module structure of certain cohomology groups (in preparation).

DEPARTMENT OF MATHEMATICS, TUFTS UNIVERSITY, MEDFORD, MASSACHUSETTS 02155

DEPARTMENT OF MATHEMATICS, BUCKNELL UNIVERSITY, LEWISBURG, PENNSYLVANIA 17837 